\title{
Mirror Technology Development for The International X-ray Observatory Mission
}

\section{Will Zhang}

IXO Mirror Technology Lead Scientist

X-ray Astrophysics Laboratory

NASA Goddard Space Flight Center 


\section{International X-ray Observatory (IXO)}

\section{ESA JAXA NASA}
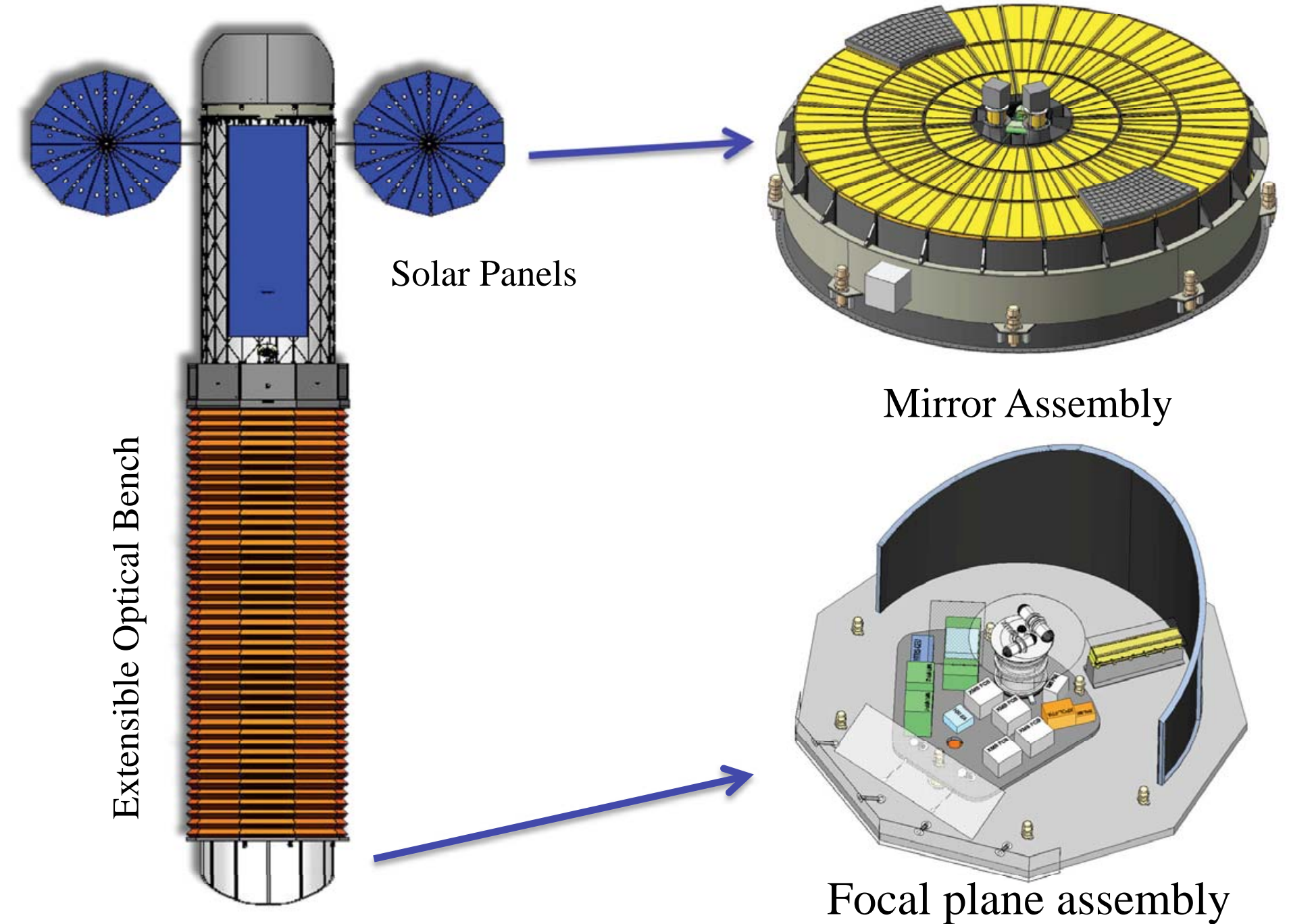

Will Zhang Mirror Tech Days

Boulder, $\mathrm{CO}$

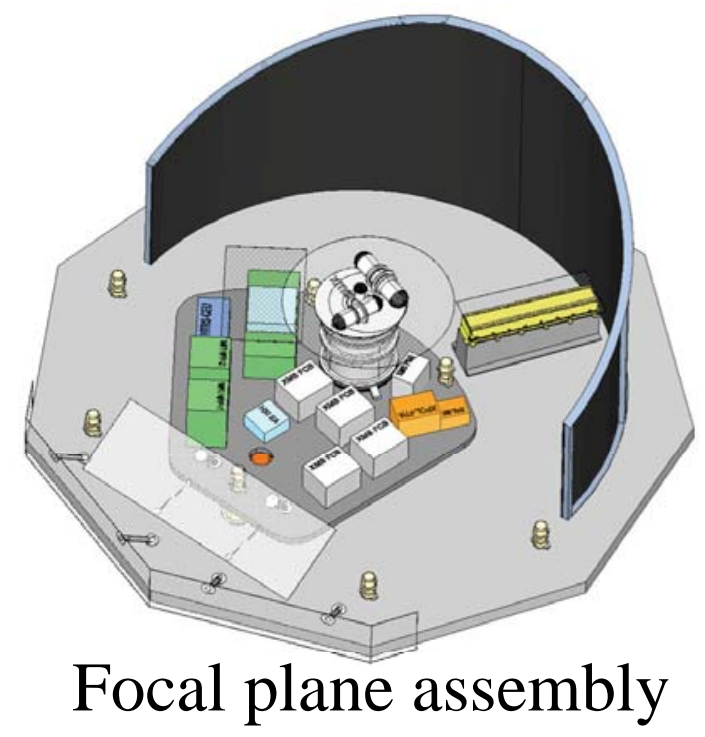

June 6, 2010 


\section{Lightweight and High Resolution X-ray Optics is Needed}

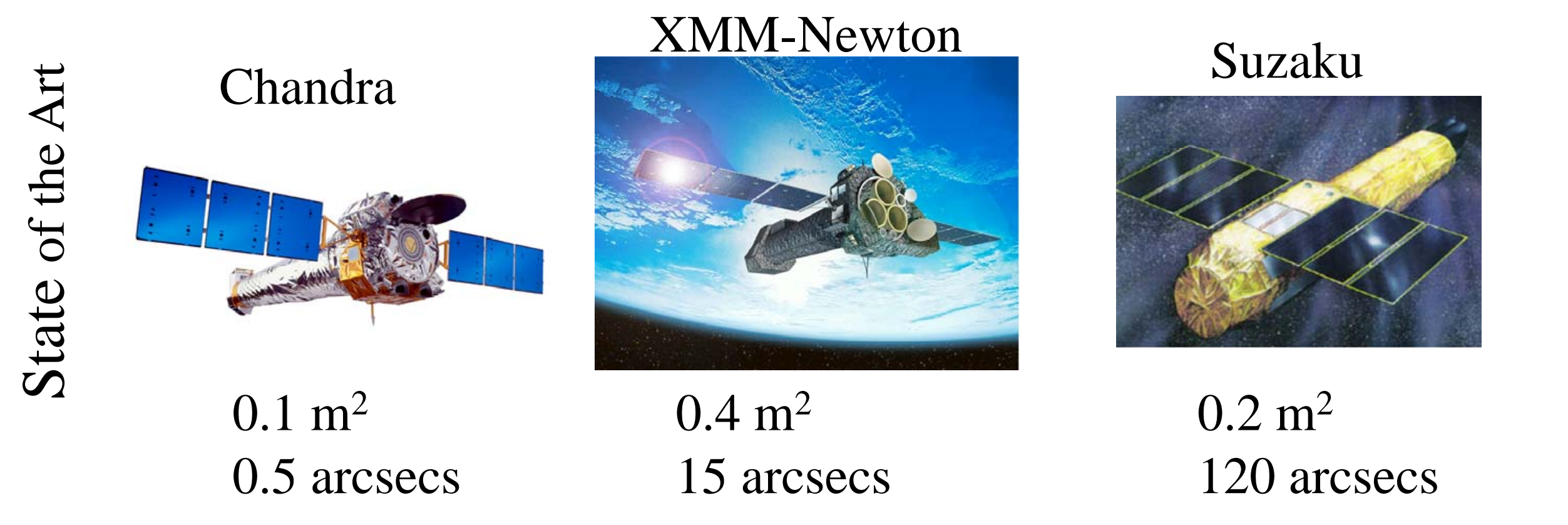

IXO Requirement 


\section{Modular Design of Mirror Assembly}

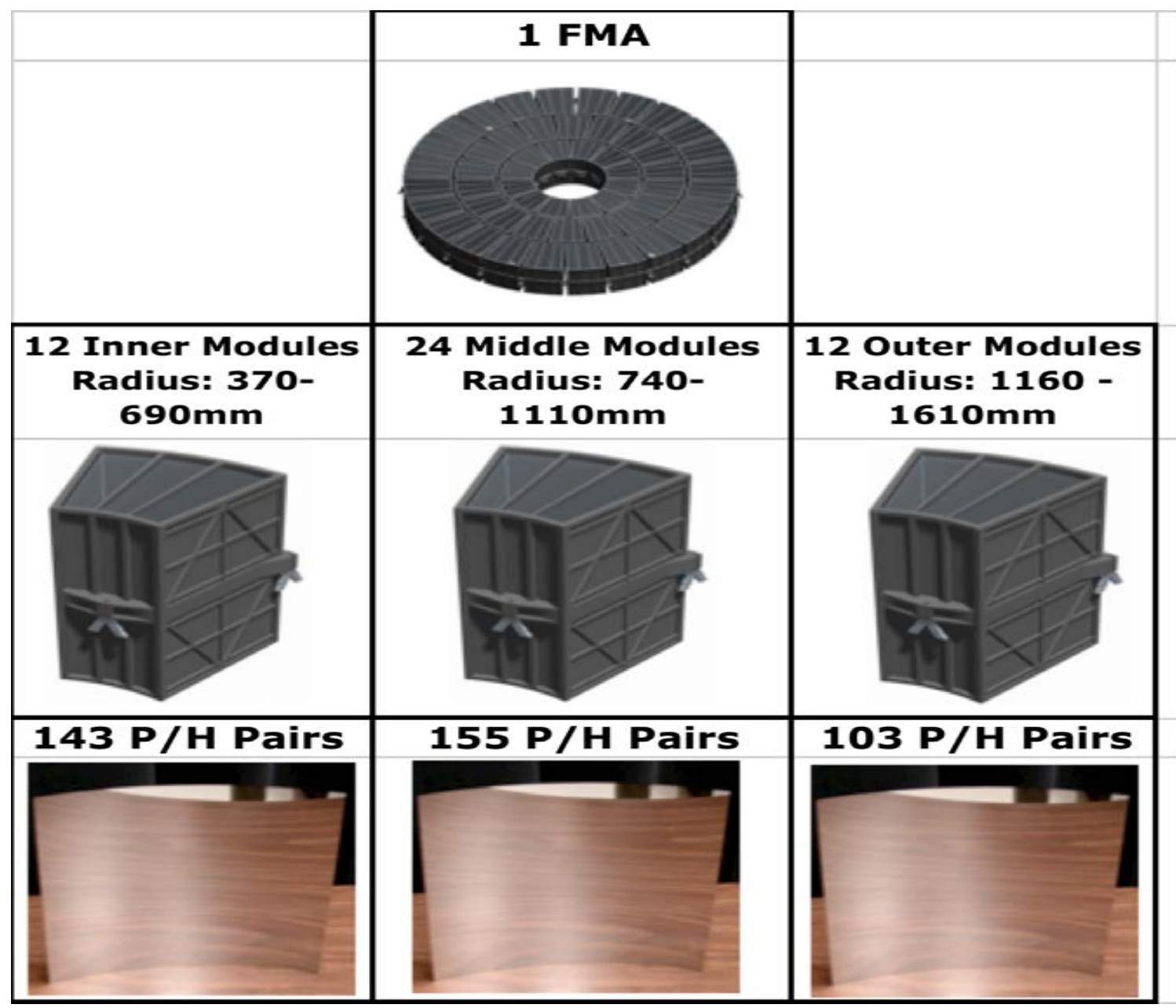

1 FMA

60
Modules

15,816

Mirror

Segments 


\section{IXO Mirror Technology Development Objectives}

- Identify problems unique to IXO mirrors that have not been encountered by, or solved for, previous missions

- Devise solutions to these problems; Demonstrate their validity through analysis and experimentation

- Establish design principles and build prototypes to prove that they meet requirements: angular resolution, effective area, mass, schedule and budget

- Subject the prototypes to X-ray and appropriate environment tests to demonstrate TRL-4, 5, and 6

Demonstrate the feasibility; Find out what's and who's out there to engineer and build the telescope! 


\section{Focus of Technology Development}

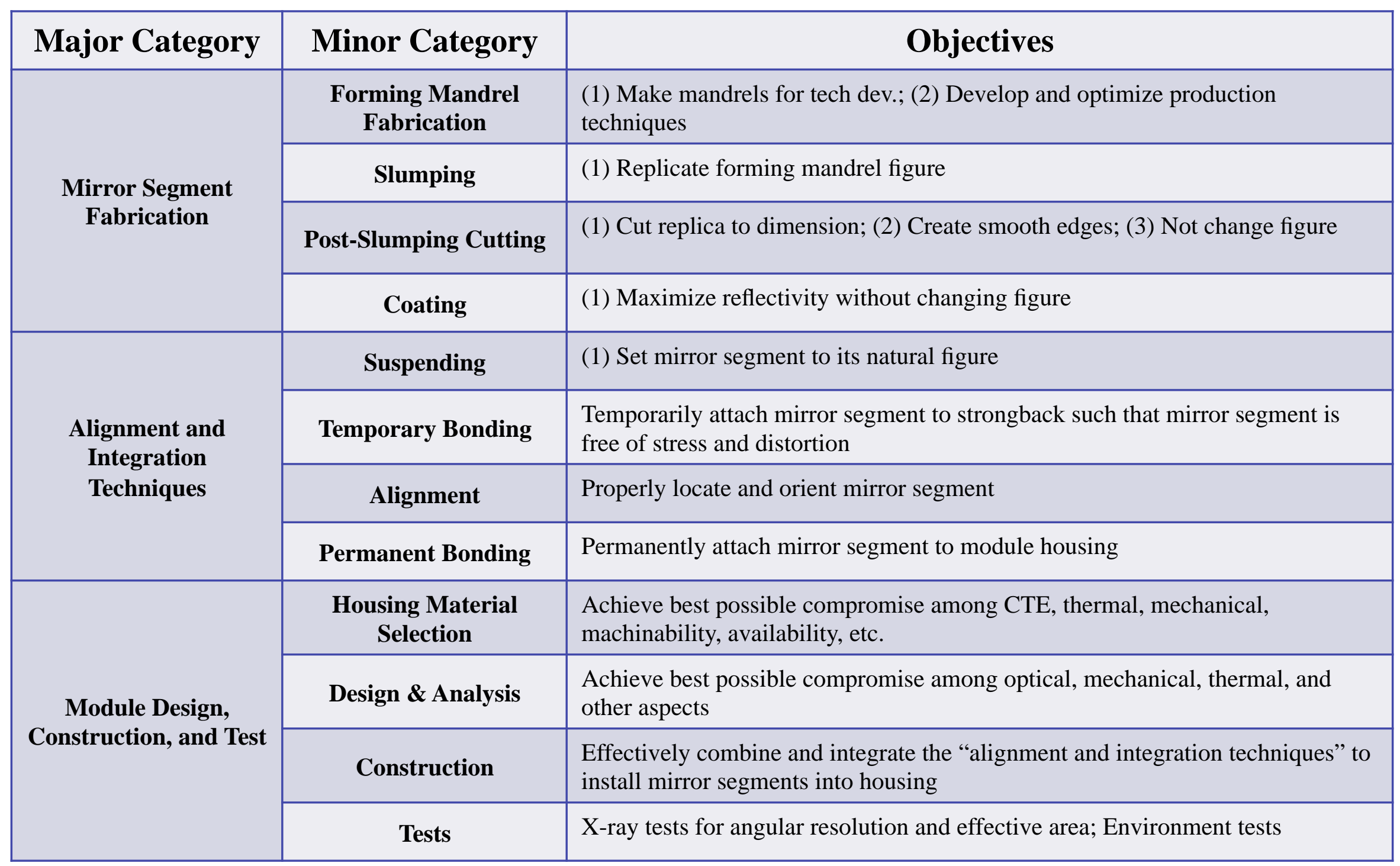




\section{Slumping - Status}

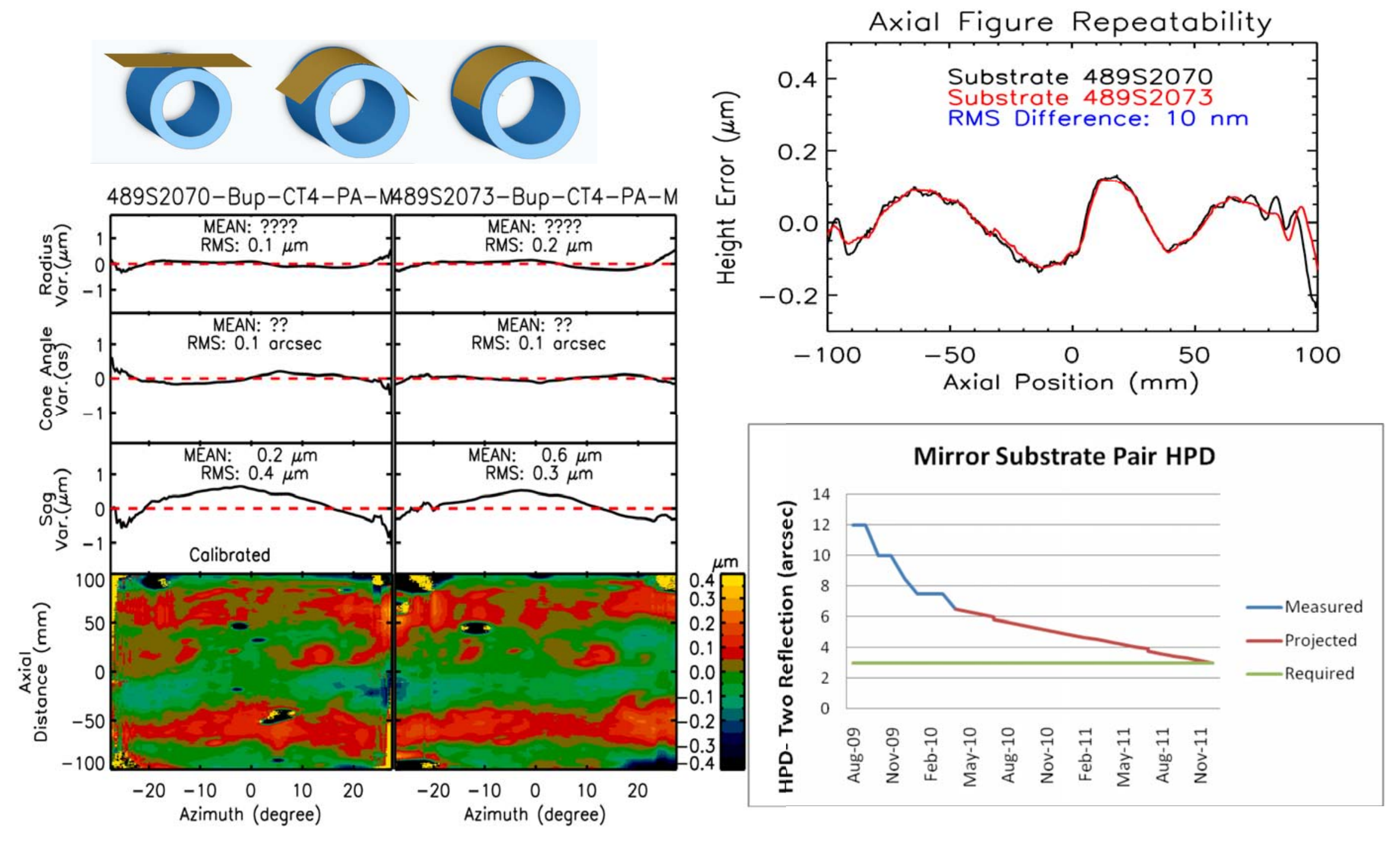

Will Zhang

Mirror Tech Days

Boulder, $\mathrm{CO}$

June 6, 2010

7 


\section{Mirror Fabrication Progress}

\begin{tabular}{|c|c|l|}
\hline Date & $\begin{array}{c}\text { HPD (two } \\
\text { reflections }\end{array}$ & \multicolumn{1}{c|}{ Comment } \\
\hline $\begin{array}{c}\text { December } \\
2008\end{array}$ & $\sim 16 "$ & $\begin{array}{l}\text { Normal incidence metrology, Full } \\
\text { illumination X-ray tests; 60-deg segments }\end{array}$ \\
\hline August 2009 & $\sim 12 "$ & $\begin{array}{l}\text { Normal incidence metrology; 60-deg } \\
\text { segments }\end{array}$ \\
\hline October 2009 & $\sim 10 "$ & $\begin{array}{l}\text { Normal incidence metrology; 30-deg } \\
\text { segments }\end{array}$ \\
\hline $\begin{array}{c}\text { December } \\
2009\end{array}$ & $\sim 8.5 "$ & $\begin{array}{l}\text { Normal incidence metrology; 30-deg } \\
\text { segments }\end{array}$ \\
\hline $\begin{array}{c}\text { January, 2010 } \\
\text { April, 2010 }\end{array}$ & $\sim 7.5 "$ & $\begin{array}{l}\text { Normal incidence metrology; 30-deg } \\
\text { segments }\end{array}$ \\
\hline $\begin{array}{c}\text { December } \\
2011\end{array}$ & $\sim 6.5 "$ & $\begin{array}{l}\text { Normal incidence metrology; 30-deg } \\
\text { segments, Using IXO mandrels }\end{array}$ \\
\hline
\end{tabular}

Will Zhang

Mirror Tech Days

Boulder, $\mathrm{CO}$

June 6, 2010

8 


\section{Temporary Bonding - Status}

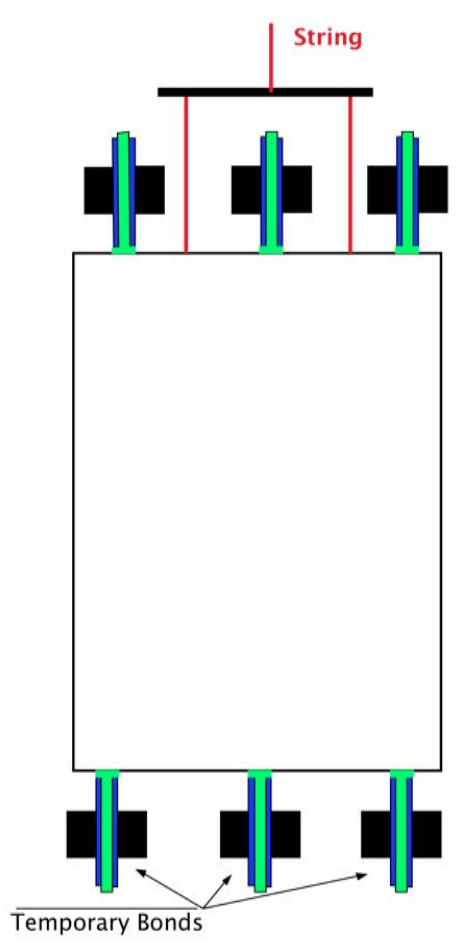

Face-On View

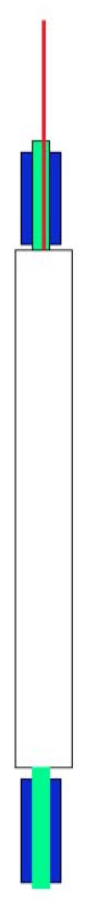

Edge-On View

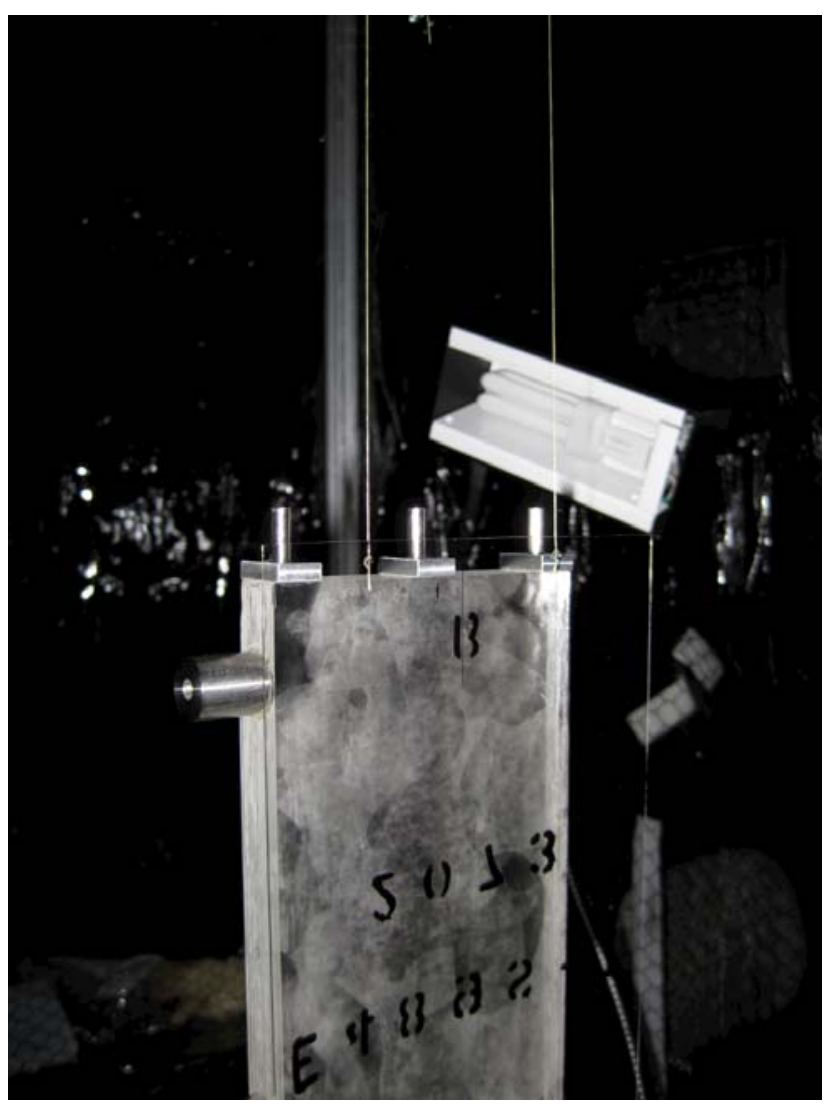




\section{Temporary Bonding - Status}

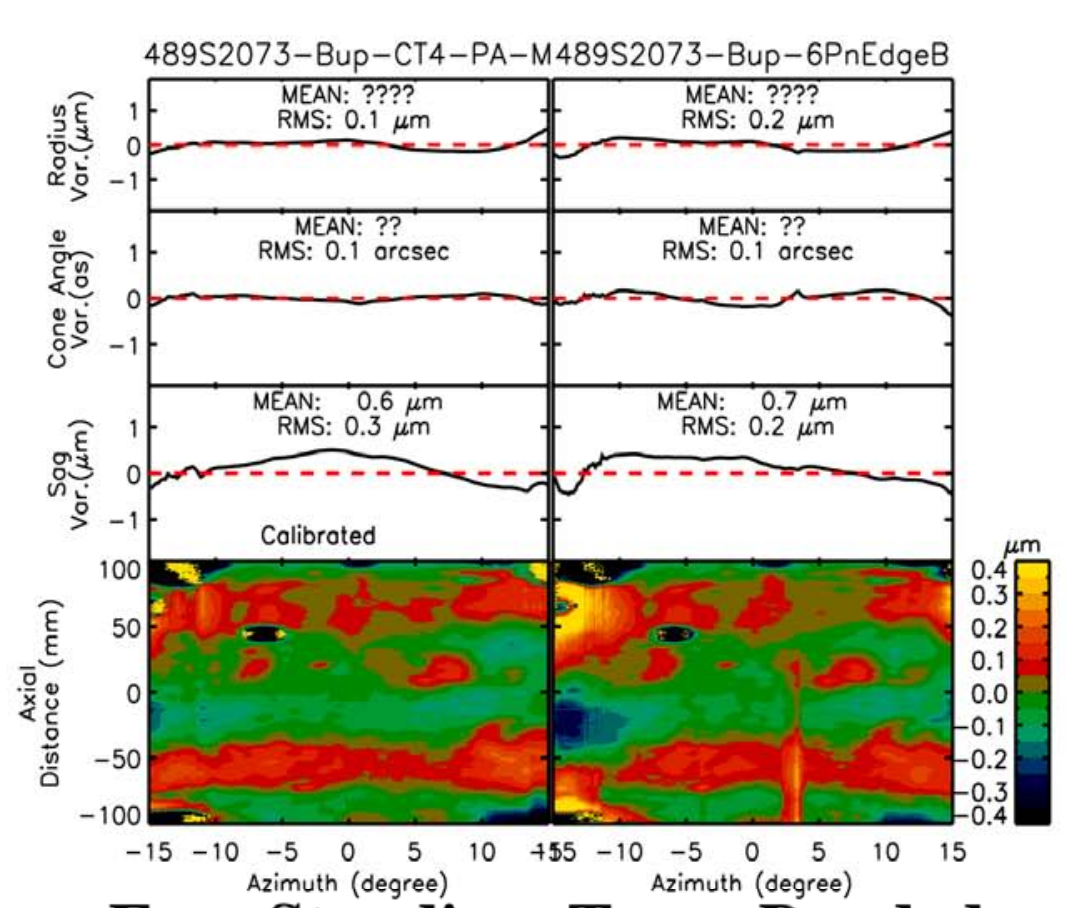

Free-Standing Temp-Bonded

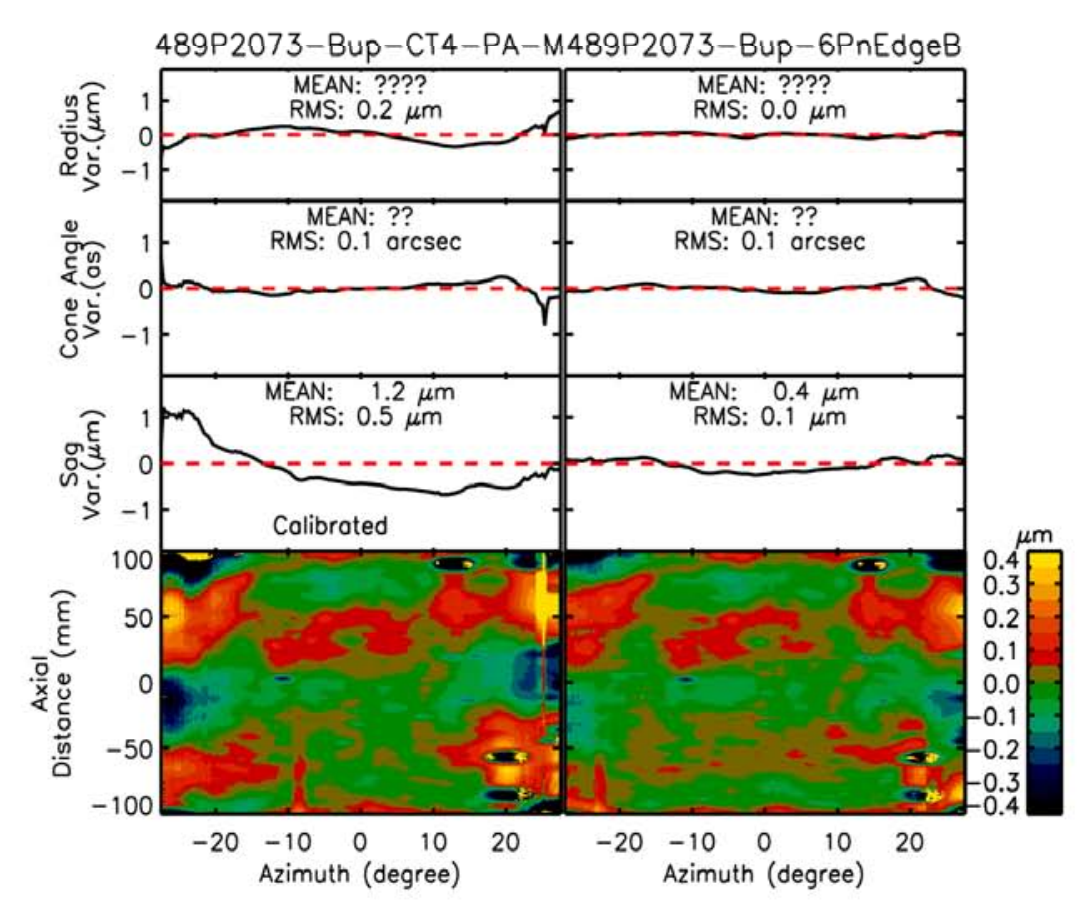

Free-Standing Temp-Bonded

- The temporary bonding process probably has met requirements, at least for smaller mirrors

- More detailed and quantitative analysis is underway

- Need to conduct experimentation with big mirror segments 


\section{Alignment - Status}
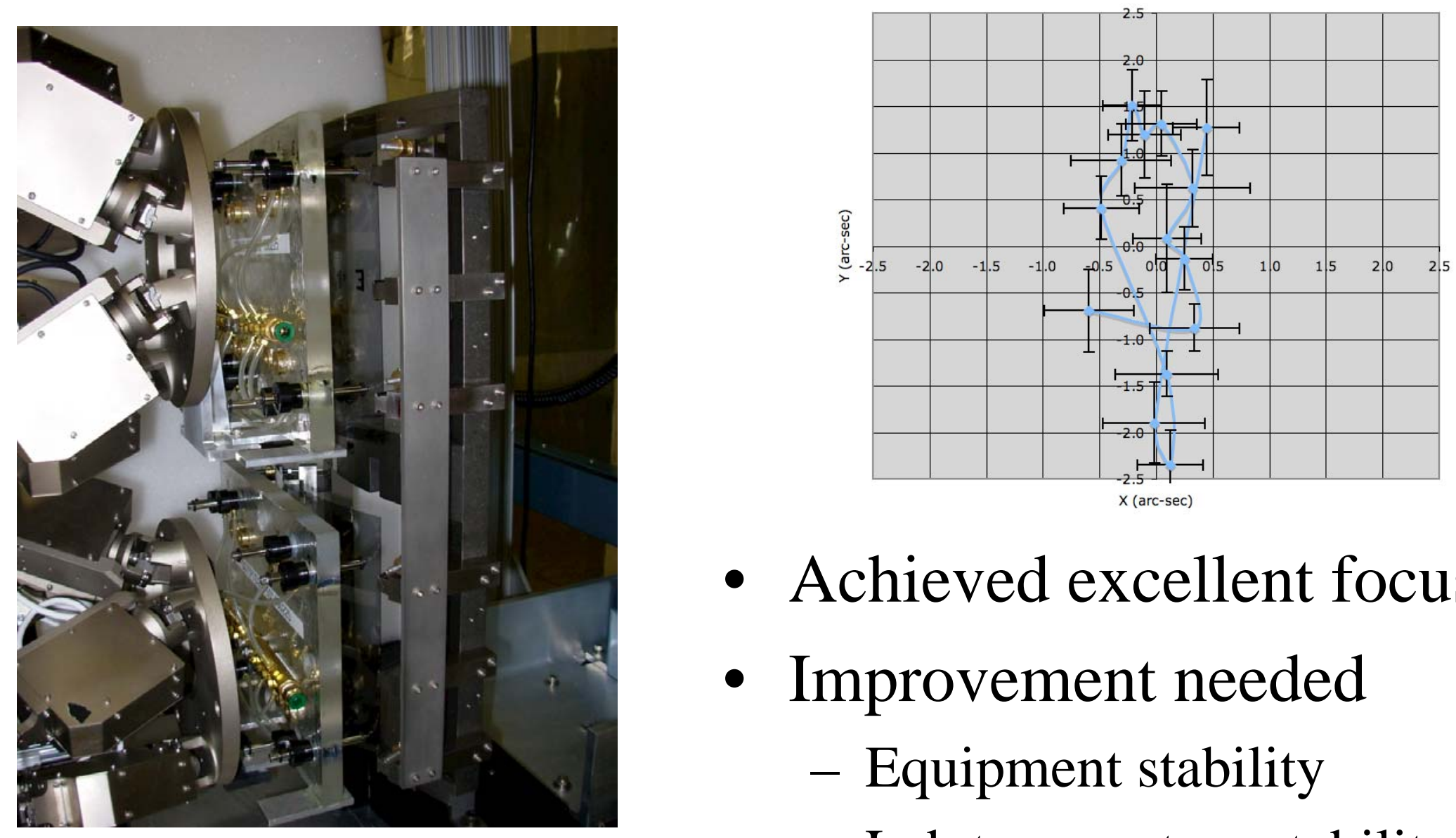

- Achieved excellent focus

- Improvement needed

- Equipment stability

- Lab temperature stability 


\section{Permanent Bonding - Status}
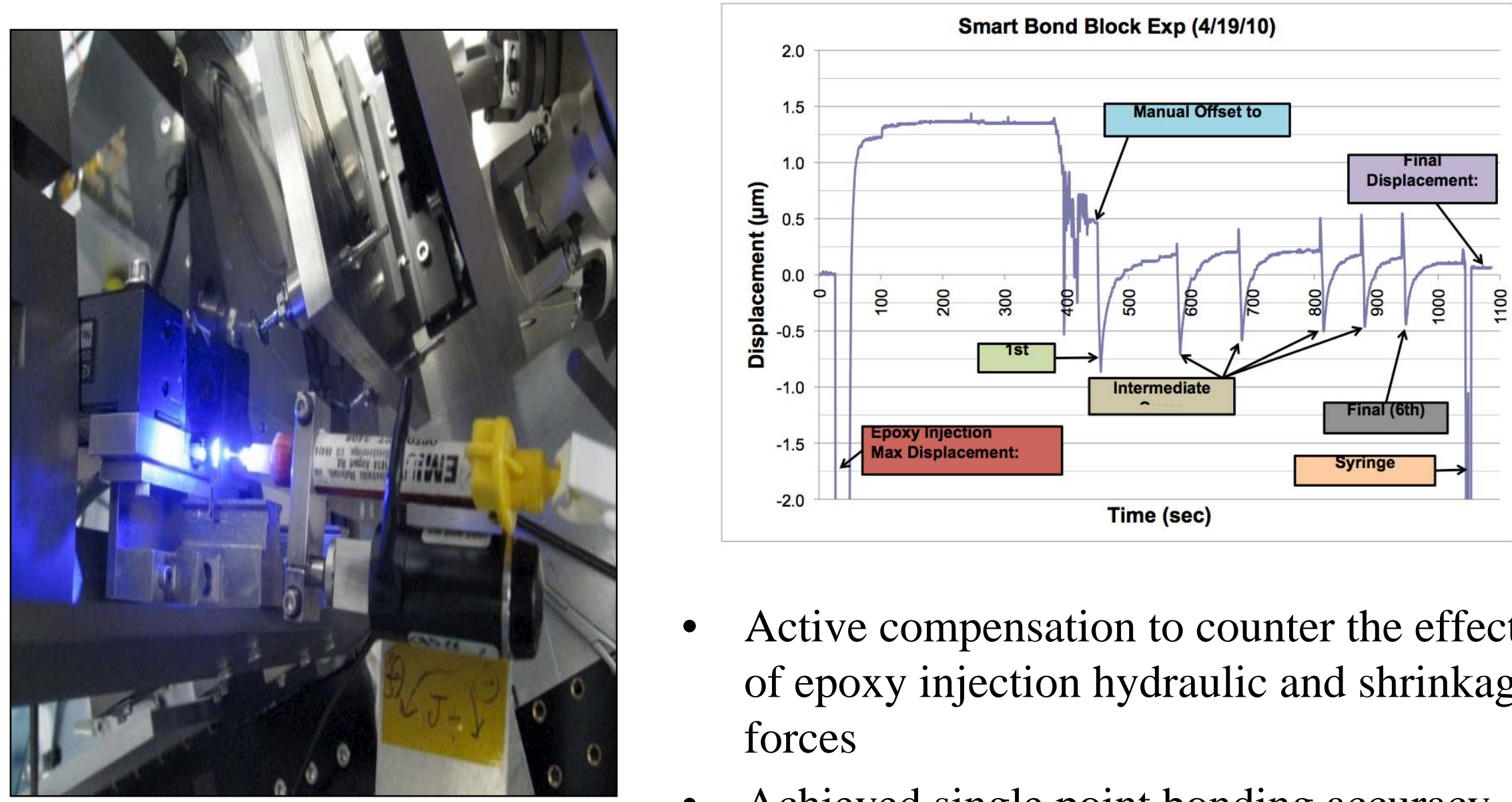

- Active compensation to counter the effects of epoxy injection hydraulic and shrinkage forces

- Achieved single point bonding accuracy of $0.1 \mu \mathrm{m}$, meeting requirements 


\section{Mirror Housing Simulator (MHS) - TRL-4}

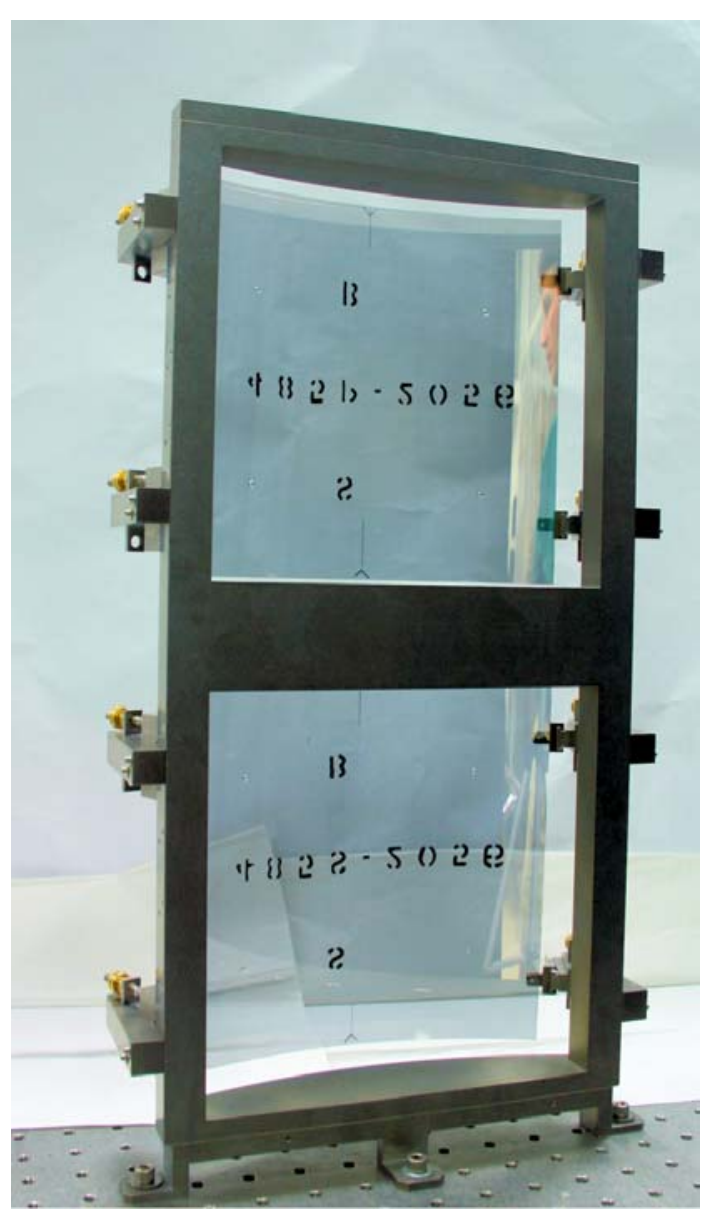

- Designed and fabricated to hold one pair of mirror segments

- Fully open and accessible to facilitate alignment, bonding, and metrology verification 


\section{Mini-Module (TRL-5)}

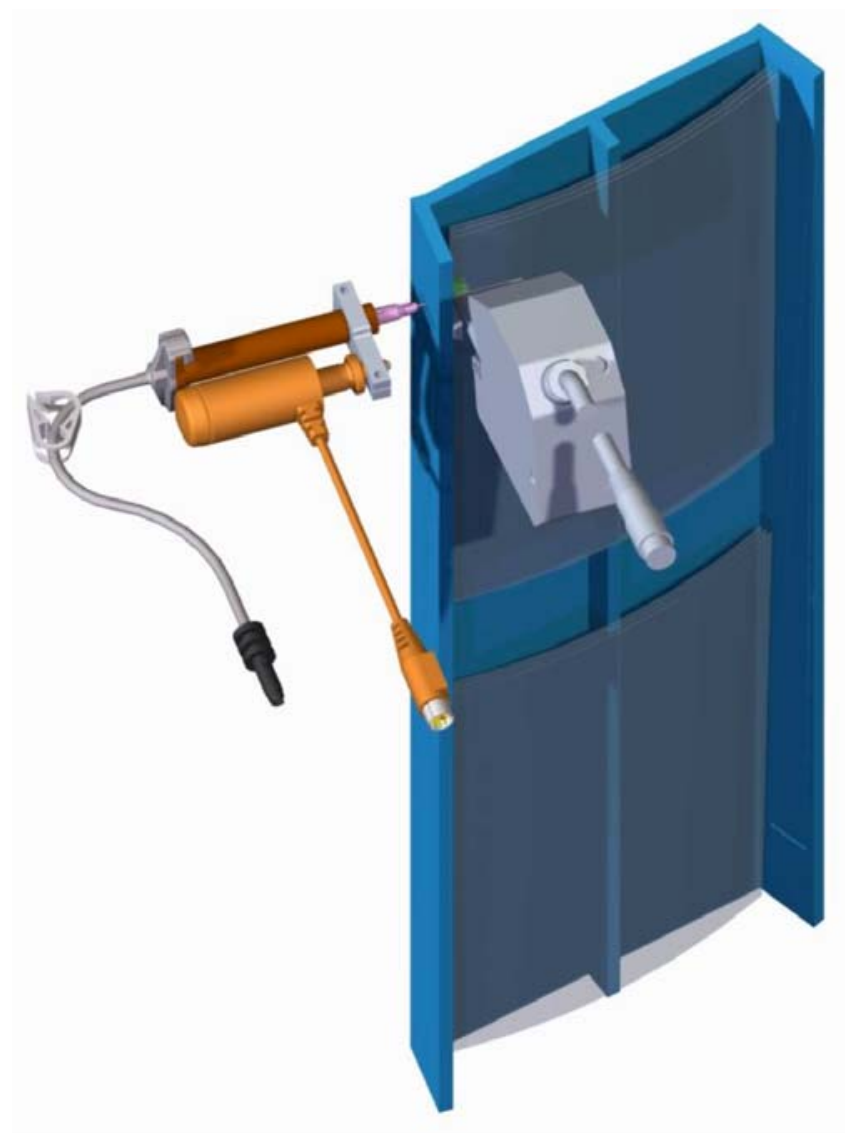

- Capable of handling multiple shells, fully testing the entire process of installing mirror segments into a module

- Capable of undergoing a full battery of tests, performance as well as environment 


\section{Flight-Like Module (TRL-6)}

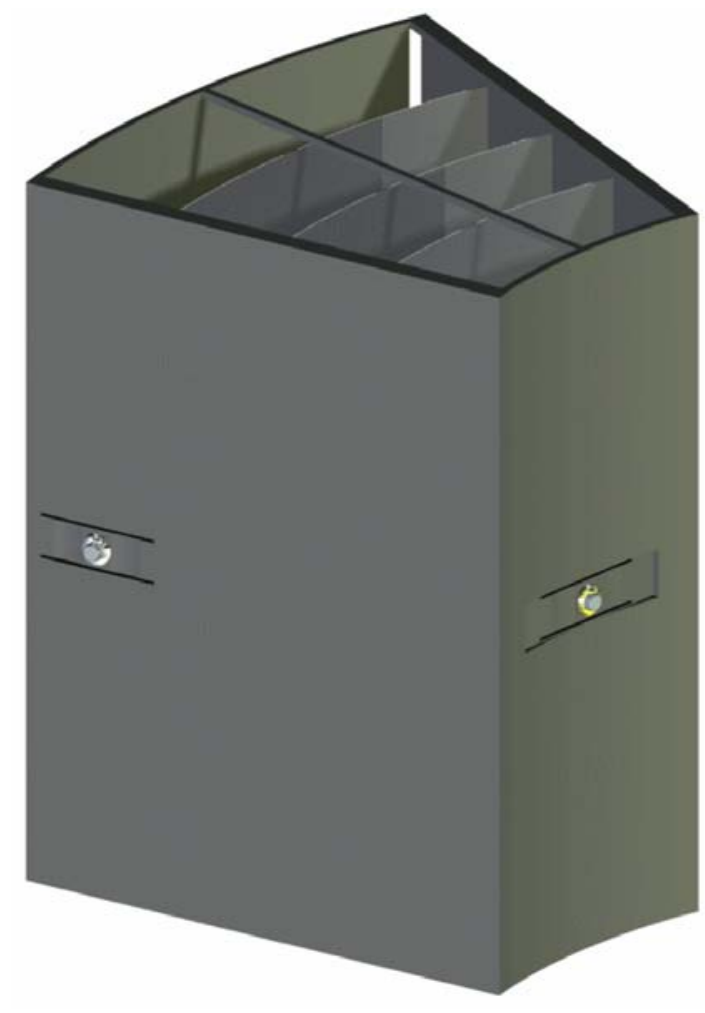

- Fully flight-like in every aspect

- Populated with both real mirror segments and mass dummies

- Will undergo a full battery of tests: X-ray, vibration, acoustic, thermal-vacuum, etc.

Angular resolution: 3.8" (half-power diameter or HPD) 


\section{Mirror Technology Development Team}

M. Biskach ${ }^{3}$, P.A. Blake, G. Byron ${ }^{3}$, K.W. Chan ${ }^{1}$, T. Evans ${ }^{3}$,

C. Fleetwood ${ }^{2}$, C. $\mathrm{He}^{2}$, M. Hill, M. Hong ${ }^{3}$, Lalit Jalota ${ }^{1}$,

L. Kolos, J.M. Mazzarella ${ }^{3}$, R. McClelland ${ }^{3}$, L. Olsen ${ }^{3}$, R. Petre,

D. Robinson, T.T. Saha, M. Sharpe ${ }^{3}$, W.W. Zhang

NASA Goddard Space Flight Center

${ }^{1}$ University of Maryland, Baltimore County

${ }^{2}$ Ball Aerospace and Technologies Corp.

${ }^{3}$ Stinger Ghaffarian Technologies, Inc.

M.V. Gubarev, W.D. Jones, T. Kester, S.L. O’Dell NASA Marshall Space Flight Center

D. Caldwell, W. Davis, M. Freeman, W. Podgorski, P.B. Reid,

S. Romaine

Smithsonian Astrophysical Observatory 


\section{Outlook}

- $\quad$ Mirror fabrication milestones

- Consistent at 5" HPD (two reflections) by December 2010

- Consistently meeting requirements ( 3" HPD two reflections) by December 2011

- Improvement of metrology to identify and isolate sources of error

- Metrology mount

- Upgrade null lens

- Check for systematic effects

- Cross-check figure quality using both normal and grazing incidence measurements

- $\quad$ Suspension Mount, Alignment, and Transfer (SMAAT)

- Perfect and understand edge-bonding ( December 2010 )

- Streamline and upgrade the alignment setup to improve thermal and structural stability (December 2010)

- Transfer and bond single pairs of mirrors in mirror housing simulator (MHS) to achieve TRL-4 ( July 2010 )

- Co-align and transfer and bond multiple mirror pairs to achieve TRL-5 (May 2011)

- Module

- Housing material selection by December 2010

- Design, analysis, and partial tests in 2011

- Full TRL-6 by November 2012 


\title{
Small Technology Firms that Have Made Direct Contributions to IXO Mirror Technology Development
}

\author{
4D Technology, Tucson, AZ \\ Optimax Systems, Inc., Ontario, NY \\ QED Technologies, Rochester, NY \\ Rodriguez Precision Optics, Gonzales, LA \\ Dallas Optical Systems, Inc., Rockwall, TX \\ RAPT Industries, Inc., Freemont, CA \\ Reflective X-ray Optics LLC, New York, NY
}




\title{
Acknowledgements
}

The work is supported in part by

NASA IXO Project Office

Goddard Space Flight Center Internal Research and Development Fund

\author{
A NASA Astronomy and Physics \\ Research and Analysis (APRA) Grant
}

